\title{
Feedback Control of Robot Manipulators by Using Gravity and Inertial Effects Compensation
}

\author{
Jan Cvejn \\ Faculty of Electrical Engineering and Informatics \\ University of Pardubice \\ Pardubice, Czech Republic \\ jan.cvejn@upce.cz
}

\author{
Milan Zapletal \\ Faculty of Electrical Engineering and Informatics \\ University of Pardubice \\ Pardubice, Czech Republic
}

\begin{abstract}
The paper describes an approach to the robot manipulator control based on a compensation of non-linear gravity terms and inertial terms in the robot motion equations and applying the PD or PID-type control law. While benefits of the compensation of the gravity force effects are well known, the inertial effects compensation, which seems to be more difficult to implement, brings additional enhancement of the control quality. An approximate compensation can be carried out in a relatively simple and efficient way, if the robot dynamics is represented by a mass-point model. The remaining influences can be treated as disturbances. Unlike the inverse dynamics approach, complete and precise mathematical model of the robot is not needed, which is an important advantage, since models of robots containing more than three links are usually very complex and difficult to obtain.
\end{abstract}

Keywords—robot manipulator, robot control, PID controller, robot dynamics

\section{INTRODUCTION}

From the control perspective, robot manipulators are multi-dimensional non-linear dynamic systems. Although the influence of non-linear terms in the motion equations is partially suppressed by using gears in the actuators, especially in the case of light-weight, high-velocity robot arms for manipulating purposes, the non-linear terms in the robot motion equations cannot be neglected to achieve optimal performance.

In this paper the problem of motion control in the robot joint space is discussed. The position in the joint space is described by a $n$-dimensional vector of joint coordinates. It is assumed that the trajectory of the end point of the effector defined in the robot operational space is transformed into the robot joint space by the algorithm of inverse kinematics $[1,2]$ before the motion task is performed. The motion control layer then works with the information about the robot joint positions and velocities.

There exist more approaches to the design of the robot manipulator feedback control system. A comprehensive overview can be found in [3]. The simplest approach, suitable only for low-velocity motions, works with the actuators as with velocity generators and the effects of the robot dynamics are considered as unknown disturbances. The feedback control then can be based on common PI or PID controllers. To enhance the performance, cascade configuration with additional velocity or even acceleration feedback can be used [1]. If a partial knowledge of the robot mathematical model is available, additional enhancement can be achieved by a partial compensation of the disturbances corresponding to non-linear terms in the robot motion equations [1].
More advanced robot control architectures consider actuators as torque generators [1-3]. This approach is utilized in centralized control systems, viewing the robot dynamics in full complexity as a high-order, coupled and non-linear one. The centralized methods utilize special features of the robot dynamics. In particular, the inverse dynamics method transforms the controller design problem into a decoupled linear one by means of additional interior loop. However, applicability of this approach depends on precision of the robot mathematical model, which often cannot be guaranteed due to unknown influences, such as backslashes and flexibilities in the gears or saturations of the action forces. Therefore, practical usability usually requires some extensions, guaranteeing at least closed-loop stability [1]

An alternative approach to non-linear robot control utilizes multivariable PID-type controllers, where the controller parameters are represented by square gain matrices. If the gravity effects are perfectly compensated, it has been shown that globally asymptotically stable position regulation can be realized by the PD-type controller if the controller gain matrices are positive definite [1-5]. Analogous results in the case of the PID controller under certain conditions were obtained in [6].

A goal of this paper is to point out to the fact that besides the gravity effects, it is relatively easy to express and compute inertial terms in the robot motion equations. This additional knowledge can be used to further enhance the closed-loop behaviour in the case of multivariable PD or PID-type controllers. The remaining part of the robot dynamic model, which is evaluated in a more difficult and computationally more demanding way, plays the role of a disturbance and is suppressed by the feedback control loop.

\section{The Robot Mathematical Model}

The mathematical model of a robot arm consisting of $n$ links in an open kinematic chain and moving freely in the operation space can be considered in the form [1-3]

$$
\mathbf{B}(\mathbf{q}) \ddot{\mathbf{q}}+\mathbf{C}(\mathbf{q}, \dot{\mathbf{q}}) \dot{\mathbf{q}}+\mathbf{g}(\mathbf{q})=\boldsymbol{\tau}
$$

where $\mathbf{q}$ is the vector of joint positions and $\boldsymbol{\tau}$ the vector of total generalized force effects of actuators. If $K$ and $P$ denote the total kinetic and potential energy, respectively, $\mathbf{B}(\mathbf{q})=\partial^{2} K / \partial \dot{\mathbf{q}}^{2}$ is a positive definite position-dependent inertia matrix,

$$
\mathbf{C}(\mathbf{q}, \dot{\mathbf{q}}) \dot{\mathbf{q}}=\frac{\partial^{2} K}{\partial \mathbf{q} \partial \dot{\mathbf{q}}} \dot{\mathbf{q}}-\frac{\partial K}{\partial \mathbf{q}}
$$


is a non-linear term corresponding to the effects of centrifugal and Coriolis forces and $\mathbf{g}(\mathbf{q})=\partial P / \partial \mathbf{q}$ is the vector function corresponding to the gravity-force effects. If we assume only electrical DC actuators, by neglecting the winding inductance and mechanical friction in the actuator,

$$
\mathbf{M} \approx \mathbf{K}_{u} \mathbf{u}-\mathbf{K}_{v} \mathbf{\omega}
$$

can be considered, where $\mathbf{M}$ is the vector of motor output torques, $\mathbf{u}$ the input voltages, $\boldsymbol{\omega}$ the vector of motor angular velocities and $\mathbf{K}_{u}>0, \mathbf{K}_{v}>0$ constant diagonal matrices. In principle, (3) allows using the motors as velocity generators, where the connected load is represented as a disturbance. Alternatively, the motor can play the role of a torque generator, where the term $\mathbf{K}_{v} \boldsymbol{\omega}$, corresponding to induced voltage in winding, is considered as electromagnetic friction. In this case, the motor is usually equipped with inner current feedback, which reduces the term $\mathbf{K}_{v} \boldsymbol{\omega}$ and protects from overload [1].

The total force effects of the actuator then depends on $\mathbf{u}$ as follows:

$$
\boldsymbol{\tau}=\mathbf{K}_{r}\left(\mathbf{K}_{u} \mathbf{u}-\mathbf{K}_{v} \boldsymbol{\omega}\right)-\mathbf{D} \dot{\mathbf{q}}
$$

where $\mathbf{K}_{r}>0$ and $\mathbf{D}>0$ are diagonal matrices. The term $\mathbf{D} \dot{\mathbf{q}}$ corresponds to viscous friction in bearings and gears and $\mathbf{K}_{r}$ is the mechanical gear ratio. Coulomb friction is not taken into account. Since $\dot{\mathbf{q}}=\mathbf{K}_{r}^{-1} \boldsymbol{\omega}$ by definition of $\mathbf{K}_{r}$,

$$
\boldsymbol{\tau}=\mathbf{K}_{r} \mathbf{K}_{u} \mathbf{u}-\mathbf{D}_{r} \dot{\mathbf{q}}
$$

where $\mathbf{D}_{r}=\mathbf{K}_{r} \mathbf{K}_{v} \mathbf{K}_{r}+\mathbf{D}>0$. The equation (1) can be then rewritten into the form

$$
\mathbf{B}(\mathbf{q}) \ddot{\mathbf{q}}+\tilde{\mathbf{C}}(\mathbf{q}, \dot{\mathbf{q}}) \dot{\mathbf{q}}+\mathbf{g}(\mathbf{q})=\mathbf{K u}
$$

where $\mathbf{K}=\mathbf{K}_{r} \mathbf{K}_{u}$ and $\tilde{\mathbf{C}}(\mathbf{q}, \dot{\mathbf{q}})=\mathbf{C}(\mathbf{q}, \dot{\mathbf{q}})+\mathbf{D}_{r}$.

\section{The Robot CONTROL With COMPENSATION OF GRAVITY AND INERTIAL EFFECTS}

One basic approach to the PID control of robots [1] utilizes partial knowledge of the inertia matrix $\mathbf{B}(\mathbf{q})$, which is decomposed as

$$
\mathbf{B}(\mathbf{q})=\overline{\mathbf{B}}+\Delta \mathbf{B}(\mathbf{q})
$$

where $\overline{\mathbf{B}}$ is a constant diagonal positive definite matrix, corresponding to approximate average inertial effects on individual axes. The linear decentralized model for the controller design is then in the form

$$
\overline{\mathbf{B}} \ddot{\mathbf{q}}+\mathbf{D}_{r} \dot{\mathbf{q}}=\mathbf{K u}-\mathbf{d}
$$

where

$$
\mathbf{d}=\Delta \mathbf{B}(\mathbf{q}) \ddot{\mathbf{q}}+\mathbf{C}(\mathbf{q}, \dot{\mathbf{q}}) \dot{\mathbf{q}}+\mathbf{g}(\mathbf{q})
$$

is considered as the disturbance. Since the dynamic model (8) without the disturbance is decoupled, each robot axis can be controlled separately from the others by its own PID controller. The control performance can be enhanced by computing the term $\mathbf{g}(\mathbf{q})$ in real time and adding it to the control input. However, omitting the other non-linear terms in the robot model leaves slow modes in the response, so the control performance need not be satisfactory in the case of fast motions.

If the inertia matrix $\mathbf{B}(\mathbf{q})$ can be computed in real time as well, its knowledge can be utilized to improve the control performance in the way proposed below. The model (8) is replaced by

$$
\mathbf{B}(\mathbf{q}) \ddot{\mathbf{q}}=\mathbf{K u}-\mathbf{d}
$$

where

$$
\mathbf{d}=\tilde{\mathbf{C}}(\mathbf{q}, \dot{\mathbf{q}}) \dot{\mathbf{q}}+\mathbf{g}(\mathbf{q}) .
$$

Let $\mathbf{q}_{d}$ denote the desired value of $\mathbf{q}$ and $\mathbf{e}=\mathbf{q}_{d}-\mathbf{q}$. Consider the control action in the form

$$
\mathbf{K u}=\mathbf{B}(\mathbf{q})\left(\mathbf{R}_{0} \mathbf{e}+\mathbf{R}_{1} \dot{\mathbf{e}}\right)+\mathbf{g}(\mathbf{q})
$$

where the square matrices $\mathbf{R}_{0}$ and $\mathbf{R}_{1}$ are symmetric and positive definite. The corresponding control system structure is displayed in Fig. 1.

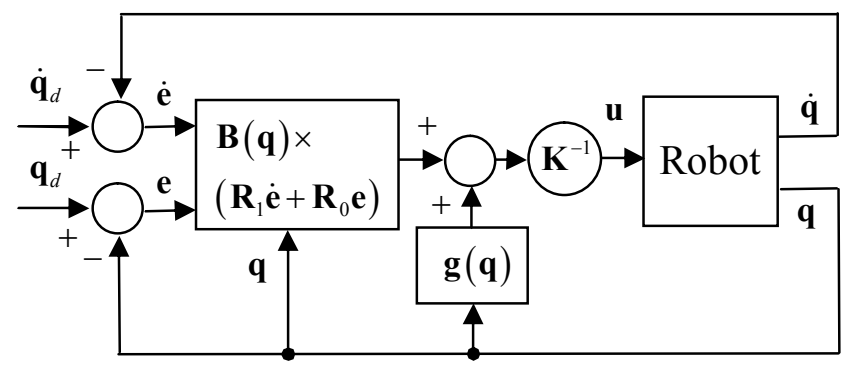

Fig. 1. The robot motion control system with the PD-type controller and compensation of $\mathbf{g}(\mathbf{q})$ and $\mathbf{B}(\mathbf{q})$

If $\dot{\mathbf{q}}_{d}=0$ is assumed, which corresponds to the position regulation problem, the error dynamics is expressed as

$$
\ddot{\mathbf{e}}+\mathbf{R}_{1} \dot{\mathbf{e}}+\mathbf{R}_{0} \mathbf{e}=\mathbf{B}^{-1}(\mathbf{q}) \tilde{\mathbf{C}}(\mathbf{q}, \dot{\mathbf{q}}) \dot{\mathbf{q}}
$$

which is equivalent to 


$$
\ddot{\mathbf{e}}+\left(\mathbf{R}_{1}+\mathbf{B}^{-1}(\mathbf{q}) \tilde{\mathbf{C}}(\mathbf{q}, \dot{\mathbf{q}})\right) \dot{\mathbf{e}}+\mathbf{R}_{0} \mathbf{e}=\mathbf{0} .
$$

If we use the Lyapunov function candidate

$$
V(\mathbf{e}, \dot{\mathbf{e}})=\frac{1}{2}\left(\dot{\mathbf{e}}^{T} \dot{\mathbf{e}}+\mathbf{e}^{T} \mathbf{R}_{0} \mathbf{e}\right)>0
$$

the time derivative of $V(\mathbf{e}, \dot{\mathbf{e}})$ along a trajectory of (13) equals

$$
\begin{aligned}
& \dot{V}(\mathbf{e}, \dot{\mathbf{e}})=\dot{\mathbf{e}}^{T} \ddot{\mathbf{e}}+\dot{\mathbf{e}}^{T} \mathbf{R}_{0} \mathbf{e}= \\
& =-\dot{\mathbf{e}}^{T}\left[\left(\mathbf{R}_{1}+\mathbf{B}^{-1}(\mathbf{q}) \tilde{\mathbf{C}}(\mathbf{q}, \dot{\mathbf{q}})\right) \dot{\mathbf{e}}+\mathbf{R}_{0} \mathbf{e}\right]+\dot{\mathbf{e}}^{T} \mathbf{R}_{0} \mathbf{e}= \\
& =-\dot{\mathbf{e}}^{T}\left(\mathbf{R}_{1}+\mathbf{B}^{-1}(\mathbf{q}) \tilde{\mathbf{C}}(\mathbf{q}, \dot{\mathbf{q}})\right) \dot{\mathbf{e}}
\end{aligned}
$$

It is possible to use the assumption that $\|\mathbf{C}(\mathbf{q}, \dot{\mathbf{q}})\| \leq C_{m}\|\dot{\mathbf{q}}\|$ and $\left\|\mathbf{B}^{-1}(\mathbf{q})\right\| \leq B_{m}$, where $C_{m}>0$ and $B_{m}>0$ are known constants [3]. Since $\dot{\mathbf{q}}=-\dot{\mathbf{e}}$ if $\dot{\mathbf{q}}_{d}=\mathbf{0}$

$$
\begin{aligned}
& \dot{V}(\mathbf{e}, \dot{\mathbf{e}})=-\dot{\mathbf{e}}^{T} \mathbf{R}_{1} \dot{\mathbf{e}}-\dot{\mathbf{e}}^{T} \mathbf{B}^{-1}(\mathbf{q}) \mathbf{D}_{r} \dot{\mathbf{e}}- \\
& -\dot{\mathbf{e}}^{T} \mathbf{B}^{-1}(\mathbf{q}) \mathbf{C}(\mathbf{q}, \dot{\mathbf{q}}) \dot{\mathbf{e}} \leq-\dot{\mathbf{e}}^{T} \mathbf{R}_{1} \dot{\mathbf{e}}+B_{m} C_{m}\|\dot{\mathbf{e}}\|^{3}
\end{aligned}
$$

By choosing the elements of $\mathbf{R}_{1}>0$ sufficiently large it is possible to ensure that there exists a region $\Omega \in R^{n} \times R^{n}$ containing the origin such that $\dot{V}(\mathbf{e}, \dot{\mathbf{e}}) \leq 0$ on $\Omega$. Consequently, the error dynamics (13) is stable in the origin. Further, $\dot{V}(\mathbf{e}, \dot{\mathbf{e}})=0$ implies $\dot{\mathbf{e}}=\mathbf{0}$ and (13) then yields $\mathbf{R}_{0} \mathbf{e}=\mathbf{0}$, so $\mathbf{e}(t)$ converges to zero by LaSalle's theorem $[7,8]$.

Since the described compensation of the terms $\mathbf{g}(\mathbf{q})$ and $\mathbf{B}(\mathbf{q})$ usually cannot be precise, in real situations the controller containing integrating component

$$
\mathbf{v}=\mathbf{B}(\mathbf{q})\left(\mathbf{R}_{0} \mathbf{e}+\mathbf{R}_{1} \dot{\mathbf{e}}+\mathbf{R}_{-1} \int_{0}^{t} \mathbf{e} d t\right)
$$

will have to be used, where $\mathbf{R}_{-1}>0$. Alternatively, to eliminate the modeling errors, adaptive approach can be utilized, as proposed e.g. in [10]. Although it seems that the analysis above cannot be directly extended for the controller (17), it can be expected that if $\mathbf{R}_{-1}$ is chosen sufficiently low with respect to $\mathbf{R}_{0}$ and $\mathbf{R}_{1}$, stability will be preserved. The modeling errors of $\mathbf{B}(\mathbf{q})$ have no influence on precision of achieving the target positions, but affect the control performance. Nevertheless, it can be easily seen from (16) that even in the cases of imperfect modeling of $\mathbf{B}(\mathbf{q})$ the convergence of $\mathbf{e}(t)$ to zero can be achieved by choosing elements of $\mathbf{R}_{1}$ sufficiently large.

\section{Computation Of $\mathbf{B}(\mathbf{q})$ And $\mathbf{g}(\mathbf{q})$}

The described approach to robot control relies on computation of the terms $\mathbf{B}(\mathbf{q})$ and $\mathbf{g}(\mathbf{q})$ in (6) in real time. If the PID controller (17) is used, these terms can be computed from an approximate mathematical model of a robot. Precise mathematical model is often not available. Especially for robot manipulators with more than three links the model is usually very complex and even real-time evaluation of $\mathbf{B}(\mathbf{q})$ and $\mathbf{g}(\mathbf{q})$ can be a problem, although efficient algorithms based on Newton-Euler formulation of the robot dynamics have been proposed in literature $[9,1]$.

In many cases a simplified model can be obtained by replacing the robot links by a set of mass points. Assume that $k$-th link contains the points $\mathbf{p}_{k 1}, \ldots, \mathbf{p}_{k, n_{k}}$ with the weights $m_{k 1}, \ldots, m_{k, n_{k}}$, where the positions $\mathbf{p}_{k j}$ are defined in the $k$-th link coordinate frame. In ideal situation, the points are chosen so that $\sum_{j=1}^{n_{k}} m_{k j}$ equals the $k$-th link weight and $\sum_{j=1}^{n_{k}} m_{k j} x_{k j}^{l}, \quad \sum_{j=1}^{n_{k}} m_{k j} y_{k j}^{l}$ and $\sum_{j=1}^{n_{k}} m_{k j} z_{k j}^{l}$ for $l=1,2$ correspond to the first and second-order moments of the $k$-th link.

If $\mathbf{r}_{k}$ denotes the position of the $k$-th link origin in the $(k-1)$-th link coordinate frame and $\mathbf{T}_{k}$ the $3 \times 3$ orthogonal rotation matrix of the $k$-th link with respect to the $(k-1)$-th link, the position of $\mathbf{p}_{k j}$ with respect to the $(k-1)$-th link frame is expressed as

$$
\mathbf{p}_{k j}^{k-1}=\mathbf{r}_{k}+\mathbf{T}_{k} \mathbf{p}_{k j} .
$$

Depending on the link type, some components of $\mathbf{r}_{k}$ and $\mathbf{T}_{k}$ are constant, the others depend on the $k$-th generalized coordinate $q_{k}$. Applying (18) recursively it is possible to obtain the positions $\mathbf{p}_{k j}$ in any $i$-th link coordinate frame, where $i<k$. In general,

$$
\mathbf{p}_{k j}^{i-1}=\mathbf{r}_{i}+\mathbf{T}_{i} \mathbf{p}_{k j}^{i}, \quad \mathbf{p}_{k j}^{k}=\mathbf{p}_{k j} .
$$

The positions with respect to the base frame are denoted $\mathbf{p}_{k j}^{0}$ in (19).

For the purposes below it is advantageous to replace the backward recursive computation of $\mathbf{p}_{k j}^{0}$. Define $\mathbf{O}_{k}^{0}$ the origin of the $k$-th link with respect to the base frame. Then by using (19) it is possible to obtain

$$
\mathbf{p}_{k j}^{0}=\mathbf{O}_{k}^{0}+\mathbf{T}_{0, k} \mathbf{p}_{k j}
$$

where $\mathbf{T}_{0, k}=\mathbf{T}_{1} \mathbf{T}_{2} \ldots \mathbf{T}_{k}, \mathbf{T}_{0,0}=\mathbf{I}$ and

$$
\mathbf{O}_{k}^{0}=\mathbf{O}_{k-1}^{0}+\mathbf{T}_{0, k-1} \mathbf{r}_{k} .
$$


If $\mathbf{p}_{k j}^{0}$ are known in dependence on $q_{1}, \ldots, q_{k}$, it is possible to express the arm potential and kinetic energy:

$$
P=\left[0,0, g_{c}\right] \sum_{k=1}^{n} \sum_{j=1}^{n_{k}} m_{k j} \mathbf{p}_{k j}^{0}, K=\frac{1}{2} \sum_{k=1}^{n} \sum_{j=1}^{n_{k}} m_{k j}\left\|\dot{\mathbf{p}}_{k j}^{0}\right\|^{2}
$$

where $g_{c}$ denotes the gravity constant. The term $\mathbf{g}(\mathbf{q})$ then can be obtained directly from

$$
\mathbf{g}^{T}(\mathbf{q})=\left[0,0, g_{c}\right] \sum_{k=1}^{n} \sum_{j=1}^{n_{k}} m_{k j} \frac{\partial \mathbf{p}_{k j}^{0}}{\partial \mathbf{q}}
$$

Further, since

$$
\left\|\dot{\mathbf{p}}_{k j}^{0}\right\|^{2}=\dot{\mathbf{q}}^{T}\left(\frac{\partial \mathbf{p}_{k j}^{0}}{\partial \mathbf{q}}\right)^{T} \frac{\partial \mathbf{p}_{k j}^{0}}{\partial \mathbf{q}} \dot{\mathbf{q}}
$$

$\mathbf{B}(\mathbf{q})$ can be obtained as

$$
\mathbf{B}(\mathbf{q})=\sum_{k=1}^{n} \sum_{j=1}^{n_{k}} m_{k j}\left(\frac{\partial \mathbf{p}_{k j}^{0}}{\partial \mathbf{q}}\right)^{T} \frac{\partial \mathbf{p}_{k j}^{0}}{\partial \mathbf{q}}
$$

To compute $\mathbf{g}(\mathbf{q})$ and $\mathbf{B}(\mathbf{q})$ using (23) and (25) it is needed to evaluate the Jacobians $\partial \mathbf{p}_{k j}^{0} / \partial \mathbf{q}$. From (20)-(21) it follows that

$$
\frac{\partial \mathbf{p}_{k j}^{0}}{\partial q_{i}}=\frac{\partial \mathbf{O}_{k}^{0}}{\partial q_{i}}+\frac{\partial \mathbf{T}_{0, k}}{\partial q_{i}} \mathbf{p}_{k j}
$$

where

$$
\begin{gathered}
\frac{\partial \mathbf{O}_{k}^{0}}{\partial q_{i}}=\frac{\partial \mathbf{O}_{k-1}^{0}}{\partial q_{i}}+\frac{\partial \mathbf{T}_{0, k-1}}{\partial q_{i}} \mathbf{r}_{k}+\mathbf{T}_{0, k-1} \frac{\partial \mathbf{r}_{k}}{\partial q_{i}} \\
\frac{\partial \mathbf{T}_{0, k}}{\partial q_{i}}=\frac{\partial \mathbf{T}_{0, k-1}}{\partial q_{i}} \mathbf{T}_{k}+\mathbf{T}_{0, k-1} \frac{\partial \mathbf{T}_{k}}{\partial q_{i}}
\end{gathered}
$$

The equations (26)-(28) can be used for efficient computation of $\mathbf{g}(\mathbf{q})$ and $\mathbf{B}(\mathbf{q})$ from (23), (25). Note that $\partial \mathbf{T}_{k} / \partial q_{i}=0$ and $\partial \mathbf{r}_{k} / \partial q_{i}=0$ if $i \neq k$. The value of $\partial \mathbf{T}_{k} / \partial q_{k}$ can be easily computed analytically, depending on the type of movement. In particular, if the $k$-th link rotates around the $z$-axis of the $(k-1)$-th link frame,

$$
\mathbf{T}_{k}=\left(\begin{array}{ccc}
\cos q_{k} & -\sin q_{k} & 0 \\
\sin q_{k} & \cos q_{k} & 0 \\
0 & 0 & 1
\end{array}\right)
$$

$$
\frac{\partial \mathbf{T}_{k}}{\partial q_{k}}=\left(\begin{array}{ccc}
-\sin q_{k} & -\cos q_{k} & 0 \\
\cos q_{k} & -\sin q_{k} & 0 \\
0 & 0 & 0
\end{array}\right) .
$$

\section{Simulation RESUlTS}

Consider the 5-DOF robot arm mass-point model in Fig. 2, where $\mathbf{q}=[\varphi, \psi, \vartheta, \eta, \varepsilon]^{T}$ is the vector of generalized coordinates, which uniquely describes the robot joint position. For simplicity, each link is represented only by single mass point. This seems to be sufficient for a demonstration here, although in real cases usually more points would be needed. The rotation around the last link axis in Fig. 2 was not considered, because it has no effect on the robot dynamics.

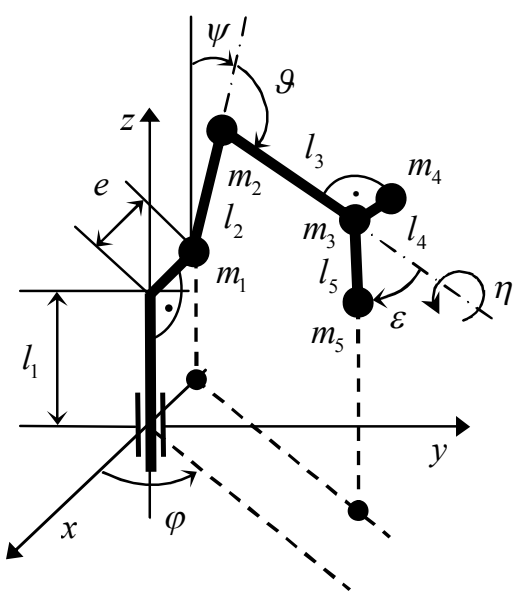

Fig. 2. The 5-DOF robot approximate model

The chosen values of the robot mechanical parameters are listed in Tab. 1.

TABLE I. THE RoBot MeCHANICAL PARAMETERS

\begin{tabular}{|c|c|c|}
\hline Parameter & Value & Unit \\
\hline$l_{1}$ & 0.5 & $m$ \\
\hline$l_{2}$ & 0.5 & $m$ \\
\hline$l_{3}$ & 0.4 & $m$ \\
\hline$l_{4}$ & 0.1 & $m$ \\
\hline$l_{5}$ & 0.3 & $m$ \\
\hline$e$ & 0.2 & $m$ \\
\hline$m_{1}$ & 2 & $\mathrm{~kg}$ \\
\hline$m_{2}$ & 1 & $\mathrm{~kg}$ \\
\hline$m_{3}$ & 1 & $\mathrm{~kg}$ \\
\hline$m_{4}$ & 0.3 & $\mathrm{~kg}$ \\
\hline$m_{5}$ & 0.7 & $\mathrm{~kg}$ \\
\hline
\end{tabular}

The terms of the diagonal matrices $\mathbf{K}_{u}, \mathbf{K}_{v}, \mathbf{K}_{r}$ in (5) were chosen as $K_{u i i}=1, K_{v i i}=0, \quad K_{r i i}=10 \quad$ and 
$\mathbf{D}=2 \times \operatorname{diag}(2,1,1,1,1)$. The joint initial and target positions are chosen as

$$
\mathbf{q}_{0}=\left[-\frac{\pi}{2}, 2, \pi, 0, \frac{\pi}{3}\right]^{T}, \mathbf{q}_{f}=\left[\frac{\pi}{2}, \frac{1}{2}, \frac{1}{10}, \pi, 0\right]^{T}
$$

First, the joint reference trajectory is chosen in the form of step function, which can be viewed as the worst-case situation, since the robot will usually track a continuous trajectory. Figure 3 shows the histories of the robot joint positions in the case of the PD controller with compensation of $\mathbf{g}(\mathbf{q})$ for $\mathbf{R}_{0}=10 \mathbf{I}$ and $\mathbf{R}_{1}=\mathbf{I}$. Figure 4 shows the histories obtained for the enhanced controller with compensation of both $\mathbf{g}(\mathbf{q})$ and $\mathbf{B}(\mathbf{q})$ for the same values of $\mathbf{R}_{0}$ and $\mathbf{R}_{1}$. It can be seen that the compensation of $\mathbf{B}(\mathbf{q})$ helped to speed up overall response and to suppress oscillations.

Figures 5 and 6 show the histories obtained for the same controller settings, but in the case of tracking the ramp-like reference trajectory

$$
q_{d i}(t)=q_{0 i}+\left(q_{f i}-q_{0 i}\right) \min \left\{\frac{t}{t_{f}}, 1\right\}
$$

with the same initial and target positions (31) as in the previous case. The time parameter $t_{f}$ in (32) was chosen as $t_{f}=1.5 \mathrm{~s}$. Although the reference trajectory is continuous, slight oscillations are visible. Faster responses that enable more precise reference tracking, but at the cost of larger magnitude of the control variable, can be achieved by using larger values of $\mathbf{R}_{0}$ and $\mathbf{R}_{1}$. Fig. 7 shows the ramp responses in the case of full compensation with $\mathbf{R}_{0}=100 \mathbf{I}$ and $\mathbf{R}_{1}=10 \mathbf{I}$.

Finally, Fig. 8 shows the histories analogous to Fig. 6, but in the case of the PID-type controller (17), where $\mathbf{R}_{0}=10 \mathbf{I}, \mathbf{R}_{1}=\mathbf{I}$ and $\mathbf{R}_{-1}=20 \mathbf{I}$. The histories in Fig. 8 are somewhat less damped than in Fig. 6, but for lower values of $\mathbf{R}_{-1}$ the differences are negligible.

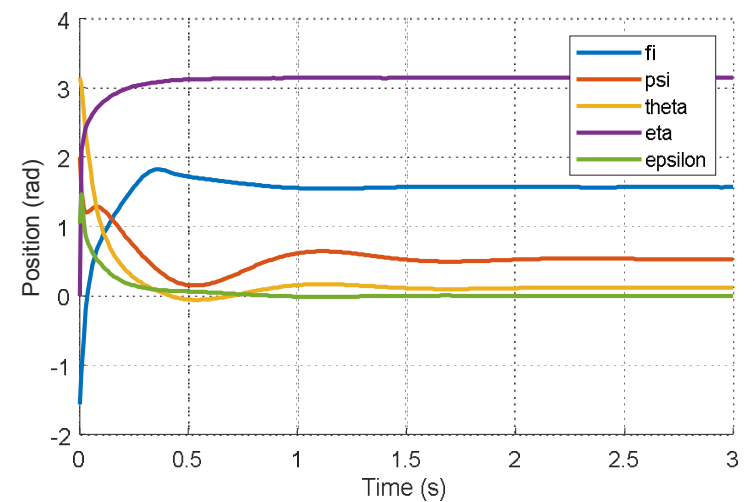

Fig. 3. The joint step responses - PD controller with compensation of $\mathbf{g}(\mathbf{q}), \mathbf{R}_{0}=10 \mathbf{I}$ and $\mathbf{R}_{1}=\mathbf{I}$

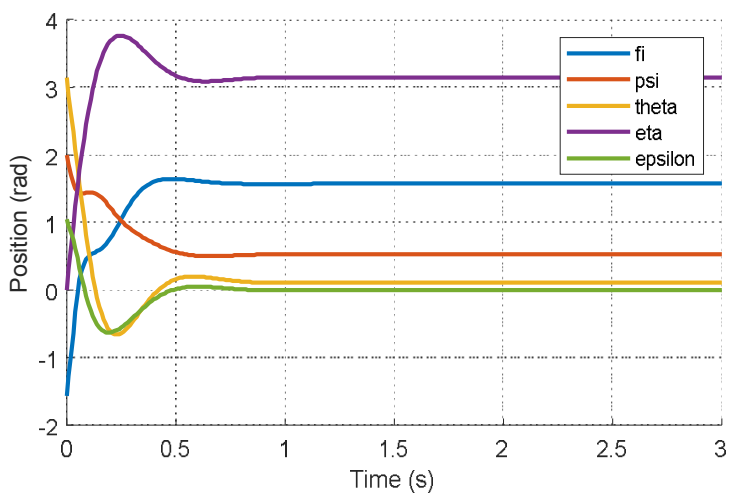

Fig. 4. The joint step responses - PD controller with compensation of $\mathbf{g}(\mathbf{q})$ and $\mathbf{B}(\mathbf{q}), \mathbf{R}_{0}=10 \mathbf{I}$ and $\mathbf{R}_{1}=\mathbf{I}$

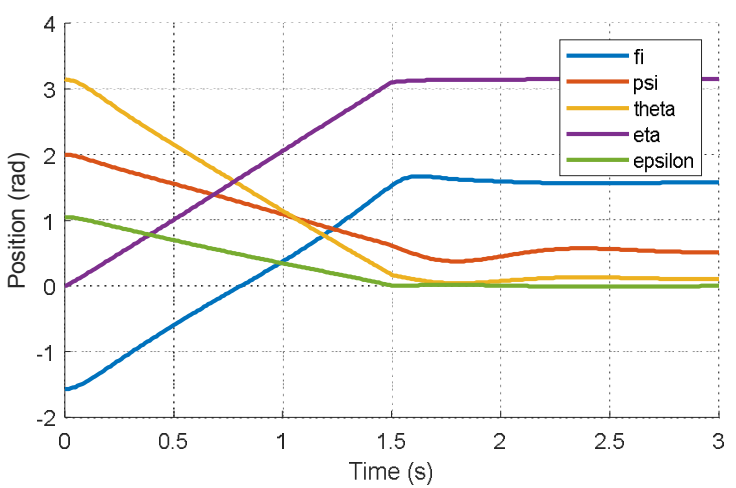

Fig. 5. The ramp responses - PD controller with compensation of $\mathbf{g}(\mathbf{q})$, $\mathbf{R}_{0}=10 \mathbf{I}$ and $\mathbf{R}_{1}=\mathbf{I}$ 


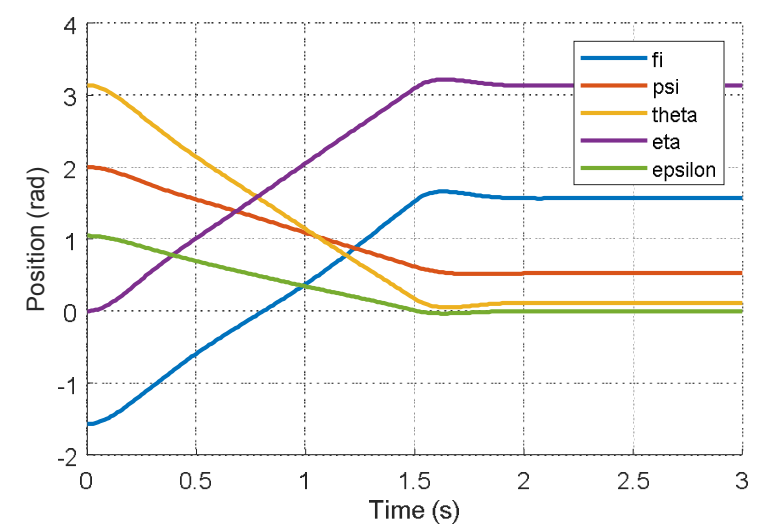

Fig. 6. The ramp responses - PD controller with compensation of $\mathbf{g}(\mathbf{q})$ and $\mathbf{B}(\mathbf{q}), \mathbf{R}_{0}=10 \mathbf{I}$ and $\mathbf{R}_{1}=\mathbf{I}$

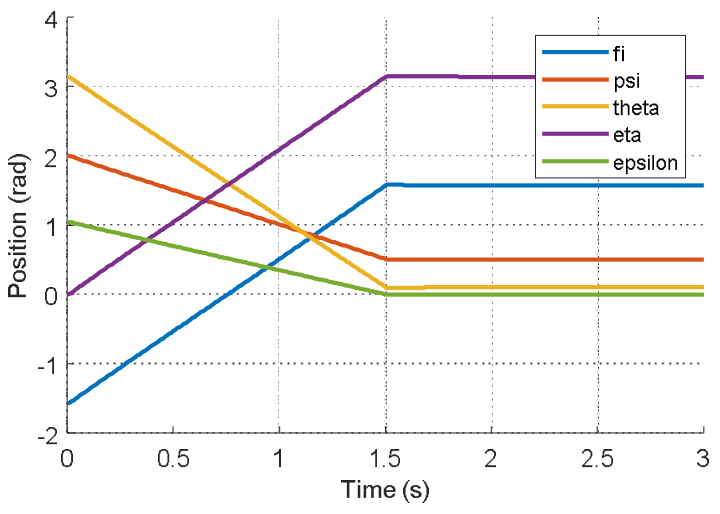

Fig. 7. The ramp responses - PD controller with compensation of $\mathbf{g}(\mathbf{q})$ and $\mathbf{B}(\mathbf{q}), \mathbf{R}_{0}=100 \mathbf{I}$ and $\mathbf{R}_{1}=10 \mathbf{I}$

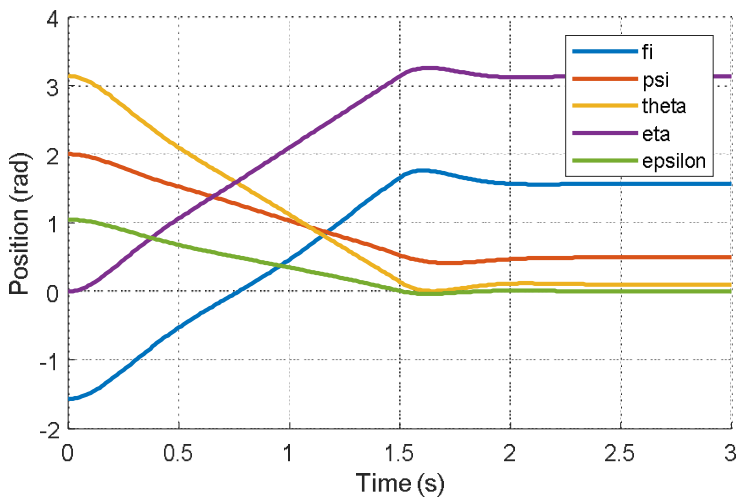

Fig. 8. The ramp responses - PID controller with compensation of $\mathbf{g}(\mathbf{q})$ and $\mathbf{B}(\mathbf{q}), \mathbf{R}_{0}=10 \mathbf{I}, \mathbf{R}_{1}=\mathbf{I}$ and $\mathbf{R}_{-1}=20 \mathbf{I}$

\section{CONCLUSIONS}

In the paper an approach to the robot manipulator control based on a compensation of the non-linear gravity and inertial terms in the robot motion equations was described. Although the gravity compensation is sufficient for global asymptotic stability of the control error in the case of the PDtype control law, additional compensation of the inertial effects enables to obtain overall faster and more damped responses for given magnitude level of the control variable. In comparison to the inverse dynamics approach, complete and precise mathematical model of the robot is not needed. Stability and convergence of the position error to zero for $\mathbf{R}_{0}>0$ and sufficiently large $\mathbf{R}_{1}>0$ has been proven for the position regulation problem, i.e. in the case of tracking the step-wise reference trajectory.

An important aspect of this approach is the way how the terms $\mathbf{B}(\mathbf{q})$ and $\mathbf{g}(\mathbf{q})$ in (6) are efficiently computed in real time, which is discussed in this paper as well. For the purposes discussed here it seems that a simplified model obtained by replacing the robot links by a set of mass points is suitable. Since the robot model is usually not precise, the terms $\mathbf{B}(\mathbf{q})$ and $\mathbf{g}(\mathbf{q})$ can be determined only approximately, so the PID-type controller (17) has to be used instead of the PD controller. The I-component makes the responses slower and less damped, but since the integrating term role consists mainly in the compensation of modeling errors, the values of $\mathbf{R}_{-1}$ in (17) can be set relatively low. In such cases it can be expected that the responses with the PID controller (17) are not much different from the PD controller case and this fact was also demonstrated by simulations.

\section{ACKNOWLEDGMENT}

The work was realized under financial support of Internal grant agency of University of Pardubice in the framework of Student grant competition.

\section{REFERENCES}

[1] B. Sicilliano, L. Sciavicco, and G. Oriollo, Robotics: Modelling, Planning and Control, Springer - Verlag, 2009.

[2] M. W. Spong, S. Hutchinson and M. Vidyasagar, Robot Modeling and Control, John Wiley \& Sons, 2006.

[3] W. Chung, L.-C. Fu and S.-H. Hsu, "Motion Control" in Springer Handbook of Robotics, Part A Robotics Foudations," B. Siciliano and O. Khatib Eds., Springer-Verlag, 2008.

[4] R. Kelly, "PD control with desired gravity compensation of robot manipulators: A review," Int. J. robot. Res, 16(5), pp. 660-672, 1997.

[5] Y. Choi and W. K. Chung, "PID trajectory tracking control for mechanical systems," Lecture notes in control and information sciences, 289, New York: Springer, 2004.

[6] S. Arimoto, F. Miyazaki, "Stability and robustness of PID feedback control for robot manipulators of sensory capability," in Robotics Research First International Symposium, M. Brady and R.P. Paul Eds., pp. 228-235, Cambridge, MA: MIT Press, 1984.

[7] H. Khalil, Nonlinear Systems, Englewood Cliffs, NJ: Prentice-Hall, 2001.

[8] H. J. Marquez, Nonlinear control systems. Analysis and design, NJ: J. Wiley \& sons, 2003.

[9] J. M. Hollerbach, "Dynamics," in Robot motion - Planning and control, Brady M. et al. Eds., pp. 51-71, Cambridge, MA: MIT Press, 1982.

[10] P. Tomei, "Adaptive PD controller for robot manipulators," IEEE Trans. Robot Autom, 7(4), pp. 565-570, 1991. 